\title{
Unusual Aneurysms of the Distal Internal Carotid Artery
}

\author{
Gary J. Redekop and Barrie Woodhurst
}

\begin{abstract}
Background: Supraclinoid carotid aneurysms have traditionally been classified according to their relation to the major carotid branches, but considerable variation exists with respect to site of origin, projection, and relationship to the skull base. Distal internal carotid aneurysms with a superior or medial projection are uncommon vascular lesions, with an unusually high incidence of operative complications. Methods: Surgical experience with five patients suffering from subarachnoid hemorrhage due to ruptured aneurysms of the dorsomedial wall of the distal internal carotid artery is presented, with emphasis on their angiographic appearance, anatomical features, and operative management. Results: All five patients underwent surgical clipping. Intra-operative rupture occurred in two cases, with avulsion of the aneurysm from the internal carotid artery in both. A third patient experienced recurrent subarachnoid hemorrhage three days after uneventful surgery, due to the clip shearing off of the parent vessel. Conclusions: Distal internal carotid aneurysms do not conform to the usual principles of aneurysm formation and are unique in their dorsomedial location unrelated to an arterial bifurcation. Although their anatomy is straightforward and exposure is not restricted by bone or dural structures of the skull base, they possess extremely fragile necks which make surgical management particularly hazardous.
\end{abstract}

\begin{abstract}
RÉSUMÉ: Anévrismes inusités de la carotide interne distale. Introduction: Les anévrismes carotidiens supraclinoïdiens ont été classifiés traditionnellement selon leur relation aux branches principales de la carotide. Cependant, il existe des variations considérables quant à leur site d'origine, de projection et à leur relation à la base du crâne. Les anévrismes de la carotide interne distale ayant une projection supérieure ou médiale sont des lésions vasculaires rares qui ont une incidence particulièrement élevée de complications opératoires. Méthodes: Nous présentons l'expérience chirurgicale de 5 patients ayant subi une hémorragie sous-arachnoïdienne due à la rupture d'un anévrisme de la paroi dorsomédiale de la carotide interne distale en mettant l'emphase sur leur aspect angiographique, leurs caractéristiques anatomiques et leur traitement chirurgical. Résultats: Les cinq patients ont subi une ligature chirurgicale. Une rupture peropératoire est survenue dans deux cas avec avulsion de l'anévrisme de la carotide interne dans les deux cas. Un troisième patient a présenté une récidive de l'hémorragie sous-arachnoïdienne trois jours après une chirurgie qui s'était bien déroulée parce que le clip s'est détaché du vaisseau mère. Conclusions: Les anévrismes de la carotide interne distale ne se conforment pas aux principes de la formation des anévrismes et sont uniques de par leur localisation dorsomédiale sans relation avec une bifurcation artérielle. Bien que leur anatomie soit simple et que leur exposition ne soit pas limitée par des structures osseuses ou durales de la base du crâne, ils ont un col très fragile qui rend le traitement chirurgical particulièrement hasardeux.
\end{abstract}

Can. J. Neurol. Sci. 1998; 25: 202-208

The internal carotid artery (ICA) between its entrance into the subarachnoid space and its terminal bifurcation has been divided into three segments, each named for a constant arterial branch. ${ }^{1}$ The ophthalmic segment extends from the origin of the ophthalmic artery to the origin of the posterior communicating artery $(\mathrm{PCoA})$; the communicating segment extends from the origin of the $\mathrm{PCOA}$ to the origin of the anterior choroidal artery (AChA); and the choroidal segment extends from the origin of the AChA to the carotid bifurcation. Each segment also gives off a series of perforating branches with relatively constant termination. Supraclinoid carotid aneurysms have traditionally been classified according to their relation to the major carotid branches, ${ }^{2}$ but considerable variation exists with respect to site of origin, projection, and relationship to the structures of the skull base. ${ }^{3-5}$

Recent publications have characterized the clinical, radio- graphic, and anatomical features of ophthalmic segment aneurysms. ${ }^{3,4}$ Superior hypophyseal aneurysms and ophthalmic aneurysms can be distinguished on the basis of their parent artery branches, relation to the anterior clinoid, projection, and relationship to the optic apparatus. Superior hypophyseal aneurysms are intimately related to the anterior clinoid process, and in most instances extensive bone removal is necessary to expose the proximal aneurysm neck. They project inferiorly and medially, beneath the optic nerve, and have been subclassified into paraclinoid and suprasellar variants based on the direction of enlargement. The microsurgical approach to these aneurysms

From the Department of Surgery, Division of Neurosurgery, University of British Columbia, Vancouver, British Columbia.

RECEIVED OCTOBER 23, 1997. ACCEPTED IN FINAL FORM MARCH 5, 1998.

Reprint requests to: Gary Redekop, 323-C, 700 West Tenth Avenue, Vancouver, British Columbia, Canada V5Z 4E5 
and their operative considerations have been well described..$^{3-5}$

Aneurysms arising distally along the internal carotid artery typically arise at the origin of the $\mathrm{PCoA}$ or $\mathrm{AChA}$, and project posterolaterally. True posteriorly projecting aneurysms arising from the inferior ICA wall have also been described. ${ }^{3,6}$ Distal ICA aneurysms with a superior or medial projection are uncommon vascular lesions, ${ }^{6-10}$ notable for the absence of obvious relationship to an arterial bifurcation, and with an unusually high incidence of operative complications. In this report, the surgical experience with five patients suffering from subarachnoid hemorrhage due to ruptured aneurysms of the dorsomedial wall of the distal internal carotid artery is discussed, with an emphasis on their angiographic appearance, anatomical features, and operative management.

\section{Clinical Material and Methods}

\section{Patient Population}

Between January, 1993 and December, 1996, 351 patients with angiographically confirmed saccular aneurysms were admitted to the neurosurgical service at the Vancouver Hospital and Health Sciences Centre. Five patients (1.4\%) were found to have a saccular aneurysm arising from the distal ICA, originating from the dorsomedial wall, and without any apparent relationship to named arterial branches or small perforating vessels. All of the patients presented with subarachnoid hemorrhage; no unruptured aneurysms at this location were found incidentally. There were no other aneurysms or vascular malformations in this group. Each patient underwent four vessel angiography, allowing characterization of the aneurysm size, origin, projection, and relation to the ICA and its branches. Details of individual patient presentation, angiographic appearance, and surgical management are presented in Table 1.

\section{Radiographic Appearance}

In each case CT scan demonstrated blood within the basal subarachnoid cisterns. Significant intraparenchymal extension was present in one patient. Angiography consistently demonstrated a saccular aneurysm, often with a relatively wide neck, arising from the distal internal carotid artery on its dorsomedial aspect. Aneurysms with a primarily medial projection could be seen well on standard anterior-posterior (AP) views, while on lateral films the aneurysm could only be identified as a shadow of double density superimposed on the ICA. Often a $30^{\circ}$ oblique angiogram best demonstrated the neck anatomy (Figure 1). Aneurysms with a greater dorsal projection were visualized well on both AP and lateral views (Figure 2).

The relation of the aneurysm neck to the PCoA on lateral angiograms was variable; in one instance it was clearly proximal, while in four the aneurysm was level with or distal to this branch. In all cases the aneurysm arose from the dorsomedial wall of the ICA, on the side opposite the posterolateral origin of the PCoA (Figure 3).

All of the aneurysms were sufficiently distal on the ICA to be free of the anterior clinoid process. It was not felt in any case that clinoid removal was necessary to obtain proximal intracranial control or to visualize the neck of the aneurysm. In some patients, there was evidence of atherosclerotic narrowing of the ipsilateral carotid in its petrous or cavernous segment. The presence of supraclinoid carotid atherosclerosis was difficult to assess angiographically because of the potential for cerebral vasospasm to confound the interpretation of irregular vessel narrowing.

\section{Surgical Management}

A standard pterional approach to the carotid and chiasmatic cisterns was used, with wide opening of the sylvian fissure. Because of initial experience with intra-operative aneurysm rupture before proximal intracranial control of the ICA was achieved, in later cases the carotid artery was exposed in the neck prior to craniotomy. The aneurysms were distal to the clinoid process, and arose from the dorsomedial wall of the ICA without relationship to the $\mathrm{PCOA}$ or $\mathrm{AChA}$, which were visualized laterally. Brief temporary occlusion of the ICA proximally

Table 1: Distal Internal Carotid Aneurysms - Anatomy and Surgical Management.

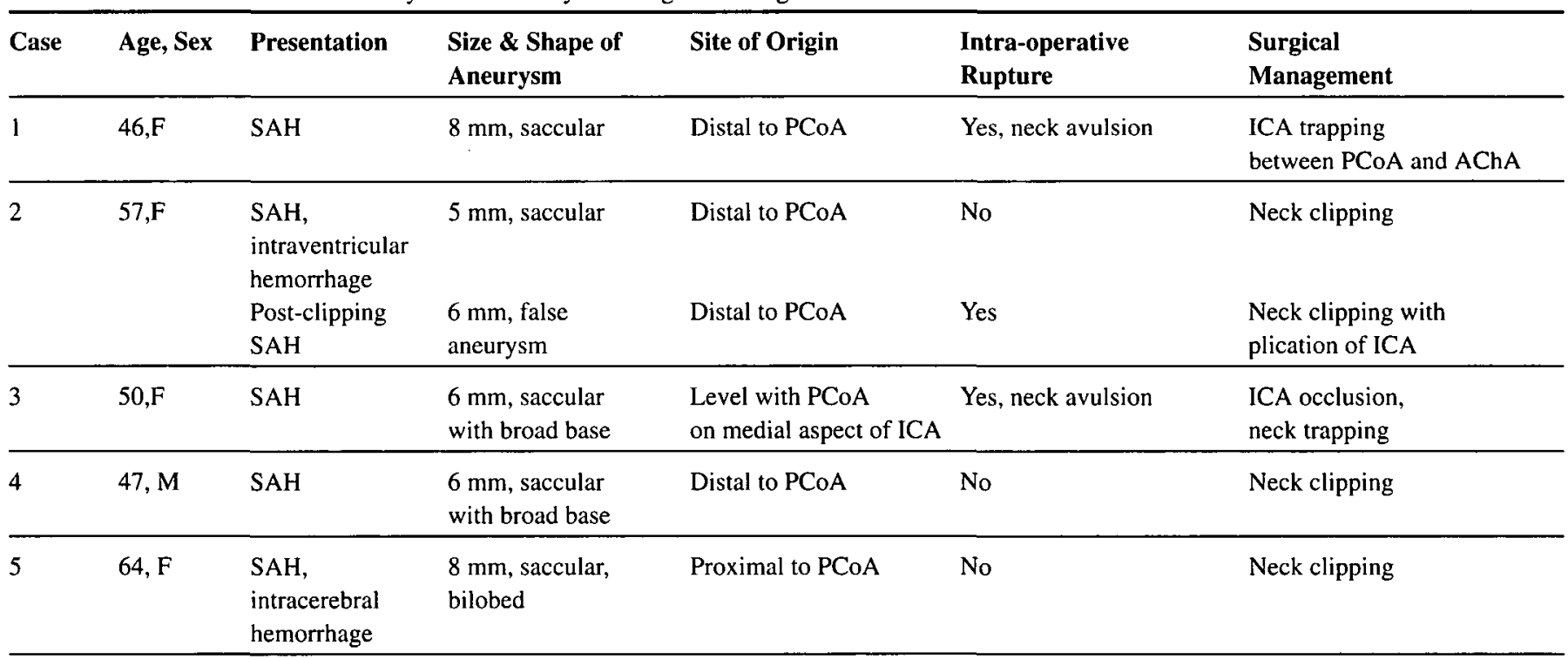



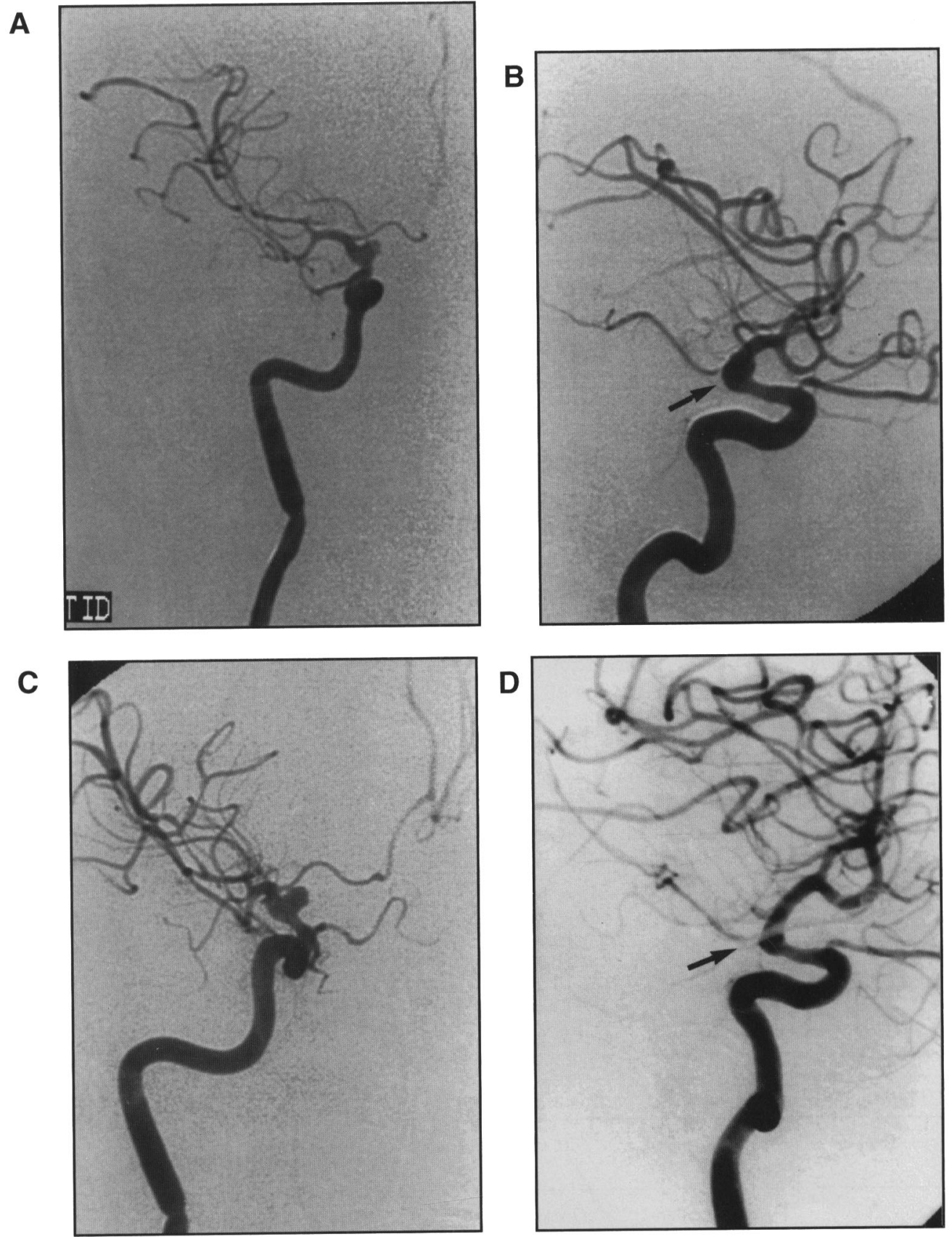

Figure I: Patient 4. Angiograms of medially pointing distal right ICA aneurysm. A small, broad-based aneurysm is seen on the AP view (A), while on the lateral view $(B)$, it is obscured by the superimposed internal carotid artery. The arrow indicates a double density corresponding to the aneurysm dome, which lies between the PCoA (not visualized) and the AChA. Optimal visualization of the aneurysm neck and anatomical relationships is obtained with a 30 degree oblique view $(C)$. After surgical clipping, the closed aneurysm clip blades demonstrate the exact location of the neck on the medial wall, just proximal to the AChA (arrow, $D$ ).

was routinely employed to reduce the turgor in the aneurysm sac at the time of clip application.

Several features of the microscopic appearance of these aneurysms were noteworthy. The wall of the carotid artery in most cases was found to be somewhat atherosclerotic, despite the relatively young age of the patients. In general, the aneurysm neck was broad relative to the size of the fundus, and the aneurysm sac and neck appeared to be very thin-walled and friable. The aneurysms were all small in size, and projected into the opticocarotid space, ${ }^{\prime}$ but there was no significant compression of the optic apparatus. There were usually several medially oriented perforating vessels arising from the proximal ICA supplying the inferior aspect of the chiasm, but no obvious relationship between the aneurysm and a perforator or the superior hypophyseal artery was convincingly established.

In three cases the aneurysm neck was clipped uneventfully, 

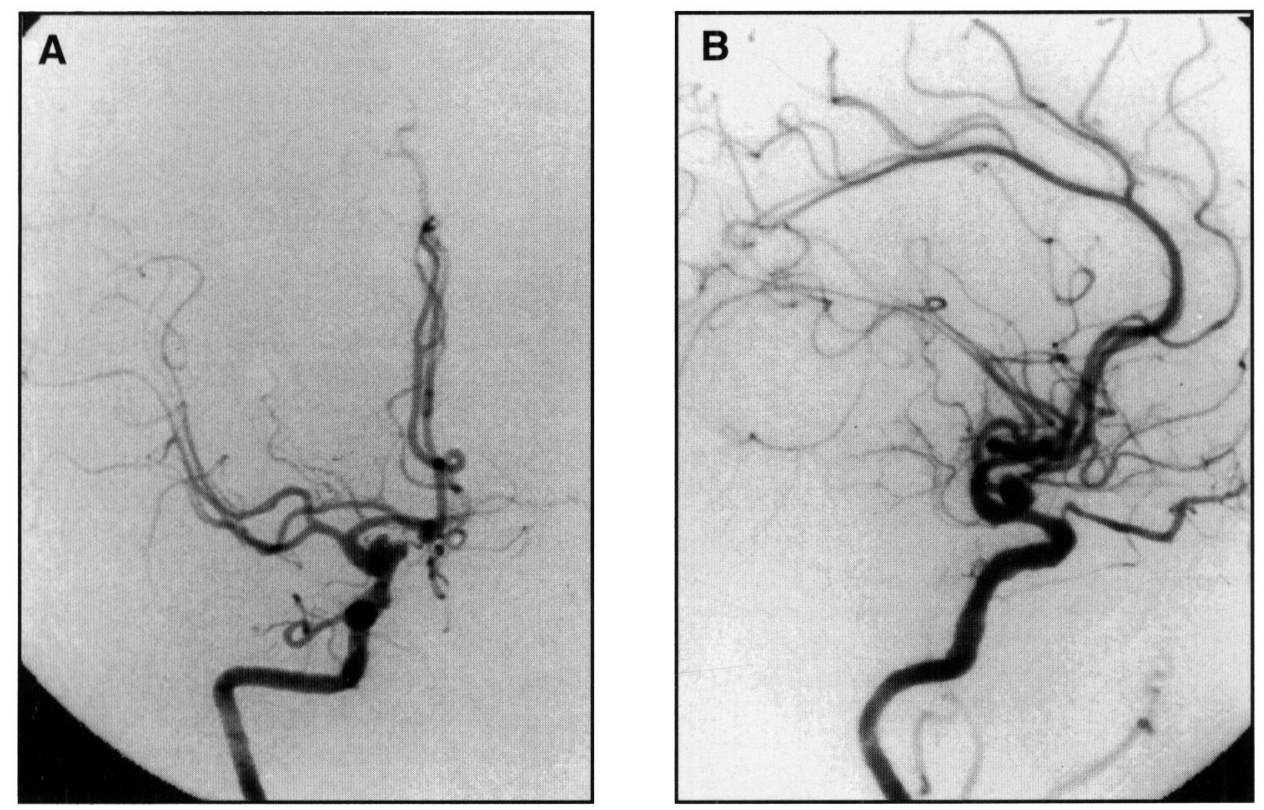

Figure 2: Patient 5. Angiograms of a dorsomedially projecting distal right ICA aneurysm. The aneurysm appears broad-based on AP view (A). The lateral view $(B)$ better demonstrates the neck, which is on the dorsomedial ICA wall, just proximal to the level of the PCoA. Incidental note is made of the accessory middle cerebral artery arising from the recurrent artery of Heubner, seen on the AP projection.

with care taken to apply the clip blades as close as possible to parallel with the carotid artery. One patient experienced recurrent SAH three days later, due to shearing off of the clip and aneurysm sac from the parent vessel. Intra-operative aneurysm rupture occurred in two cases. In both the rupture site was at the thin, friable base of the aneurysm, resulting in complete avulsion of the aneurysm from the ICA, leaving a hole several millimetres in diameter in the dorsomedial wall of the carotid artery. Three cases are described to illustrate potential hazards and surgical considerations in the management of these uncommon aneurysms.

\section{CASE REPORTS}

\section{Patient 1}

A 46-year-old female presented with subarachnoid hemorrhage. Angiography demonstrated a saccular aneurysm $8 \mathrm{~mm}$ in diameter arising from the dorsomedial wall of the left ICA distal to the PCoA (Figure 4). At surgery, the carotid and chiasmatic cisterns were packed with blood. Despite careful opening of the sylvian fissure with caution to avoid significant frontal lobe retraction, the aneurysm ruptured during the early stages of dissection of the proximal carotid artery, with a small tear near the neck. The neck and fundus were extremely thin-walled and friable. A temporary clip was placed on the ICA proximally, and the neck further defined. A straight clip was placed across the neck of the aneurysm, but as the blades were closed, the aneurysm sac tore away completely, leaving a gaping hole on the dorsomedial wall of the parent vessel. The ICA could not be repaired directly or with a clip graft, and the aneurysm neck was trapped between clips placed distal to the PCoA and proximal to the AChA. The pre-operative angiogram had demonstrated generous cross flow from right to left through the anterior communicating artery, and it was felt that augmentation of cerebral blood flow with a bypass graft was unnecessary. However, post-operatively there was a right hemiplegia due to an infarction in the distribution of the left middle cerebral artery.

\section{Patient 2}

A 57-year-old female presented with subarachnoid and intraventric- ular hemorrhage as well as acute hydrocephalus. After emergency placement of a ventriculostomy, angiography revealed a small aneurysm arising from the right ICA, projecting dorsomedially, with its origin distal to the PCoA (Figure 5, A and B). At surgery, the internal carotid artery was noted to be atherosclerotic and tortuous. The aneurysm projected dorsomedially, lateral to the optic nerve. Brief temporary clipping of the ICA proximally was used to soften the aneurysm sac and a straight clip was placed across the neck, flush with the parent vessel. No major branches or perforating vessels were related to the aneurysm neck. The aneurysm was punctured with a 25 gauge needle and collapsed.

Three days later, the patient deteriorated abruptly and CT scan revealed more subarachnoid blood, consistent with recurrent hemorrhage. Angiography showed that the aneurysm clip was displaced away from the ICA, and a new, slightly larger and more medially projecting aneurysm was apparent (Figure 5, C and D). It was unclear on the basis of the angiogram whether the new aneurysm represented a "slipped clip", regrowth of an incompletely clipped aneurysm, or avulsion of the aneurysm neck with formation of a pseudoaneurysm. Re-exploration of the aneurysm resulted in torrential hemorrhage from a false aneurysm of the internal carotid artery. The clip blades had been torn away from the parent vessel and still contained thin fragments of the collapsed aneurysm sac. The supraclinoid segment of the ICA was isolated with temporary clips and the opening of the aneurysm neck closed with 2 fenestrated right-angled clips, resulting in plication of the parent vessel to approximately 50 percent of its original caliber.

\section{Patient 4}

A 47-year-old man presented with severe headache and photophobia. CT scan demonstrated diffuse subarachnoid blood, and angiography revealed a small aneurysm of the distal right ICA, pointing superiorly and medially (Figure 1). At surgery, the aneurysm was found to have a relatively broad neck, with a distinct margin between the thick. and atherosclerotic carotid artery and the very thin aneurysm sac. To prevent a tear at the neck during clip placement, the supraclinoid segment of the ICA was trapped using temporary clips proximally and distally, as well as on the PCoA. The softened aneurysm was then obliterated using a straight clip from a slightly oblique trajectory, with an effort to stay absolutely flush with the parent artery and to incorporate some of the normal arterial wall into the clip blades without caus- 


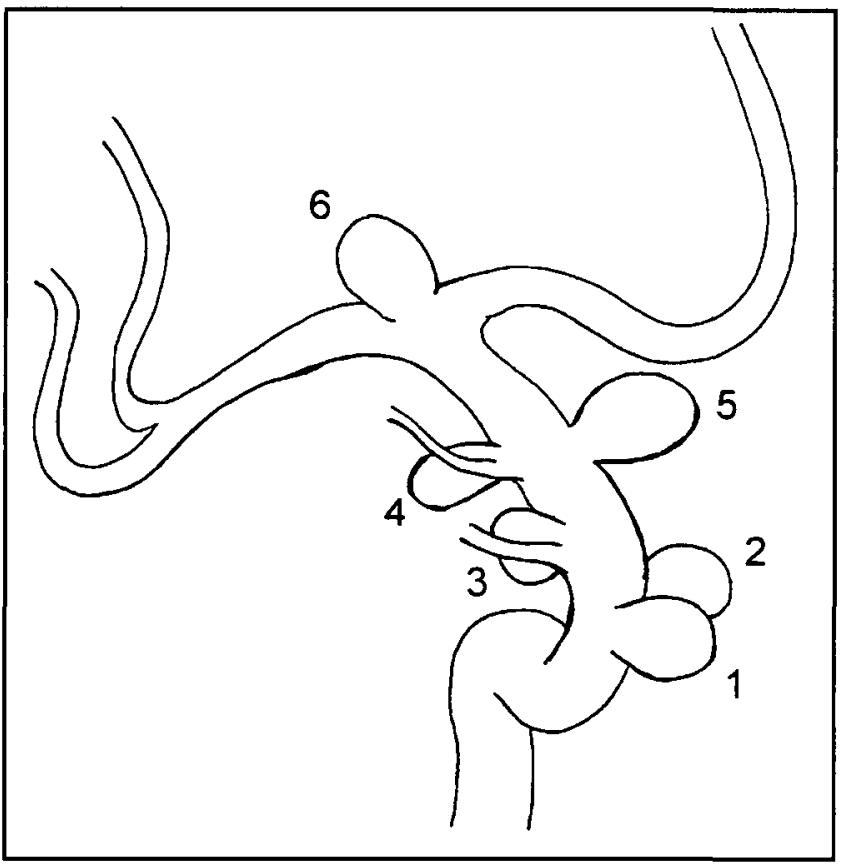

Figure 3: Line diagram demonstrating the location of these unusual aneurysms arising from the dorsomedial wall of the ICA, on the side opposite the posterolateral origin of the PCOA. Also illustrated are typical internal carotid artery aneurysm locations. $(l=$ ophthalmic aneurysm; 2 = superior hypophyseal aneurysm; 3 = posterior communicating aneurysm; 4 = anterior choroidal aneurysm; $5=$ distal type, dorsomedially projecting aneurysm; 6 = carotid termination aneurysm).

ing stenosis or telescoping of the vessel. The temporary clips were then removed, first distally and then proximally, and the aneurysm did not refill. There was no further bleeding.

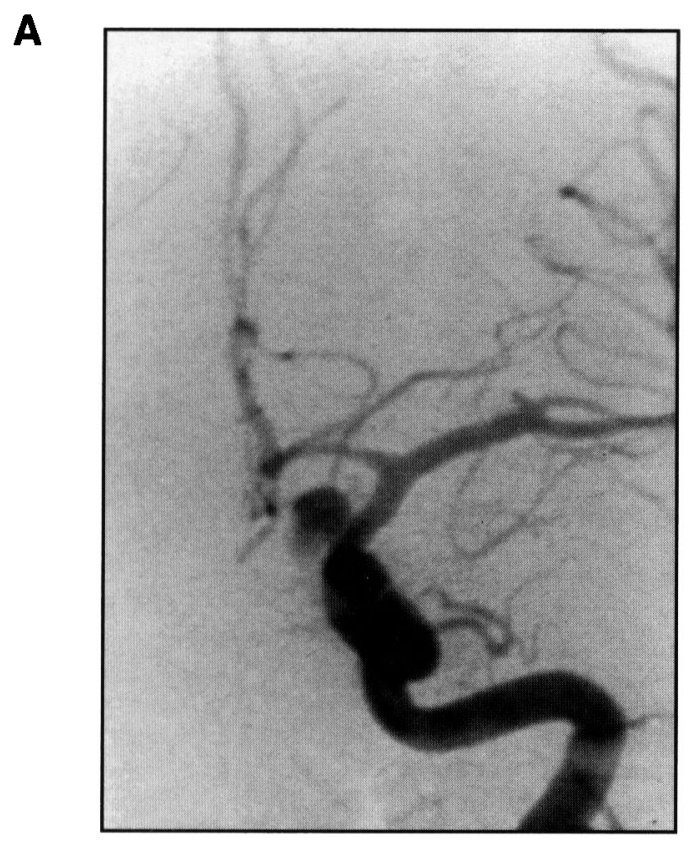

\section{Discussion}

\section{Aneurysms Unrelated to Arterial Bifurcations}

Most saccular aneurysms arise at branch points on the intracranial arterial tree. Rhoton ${ }^{2}$ has outlined the basic anatomical principles which apply to the majority of these lesions. They occur at sites of arterial branching from the parent vessel, usually at a turn or curve, and point in the direction that blood would have gone if the curve in the aneurysm were not present. Their dome or fundus points in the direction of maximal hemodynamic thrust in the pre-aneurysmal segment of the parent artery.

Aneurysms which are unrelated to arterial bifurcations are distinctly rare, but they have been described on all major cerebral vessels. ${ }^{6-11}$ The supraclinoid segment of the carotid artery is the most frequent site, but many of those reported in the literature have been so-called "blister-like" aneurysms, ${ }^{8.10}$ which are microscopic in size, thin-walled, and broad-based, without an identifiable neck. Some have been found incidentally, or seen as only subtle irregularities of the ICA wall on angiography. However, our series and cases reported by others ${ }^{6,7,10}$ suggest that there is a small group of true saccular aneurysms arising from the dorsomedial wall of the distal ICA, which do not conform to the usual anatomical principles influencing aneurysm formation. ${ }^{2}$ The incidence in this series was $1.4 \%$ of all aneurysm cases over a four year period.

Several physical factors have been shown to play a role in aneurysm formation, growth, and rupture. It is now accepted that saccular aneurysms are acquired, degenerative lesions of the cerebral arteries, forming primarily as a result of hemodynamic stress, possibly in combination with pre-existing structural defects. ${ }^{12-14}$ The axial stream impingement of blood flow at the apex of a bifurcation renders these locations particularly susceptible to aneurysm formation. ${ }^{12}$ Further outpouching of the vessel wall, turbulence within the sac, and destruction of the internal

B

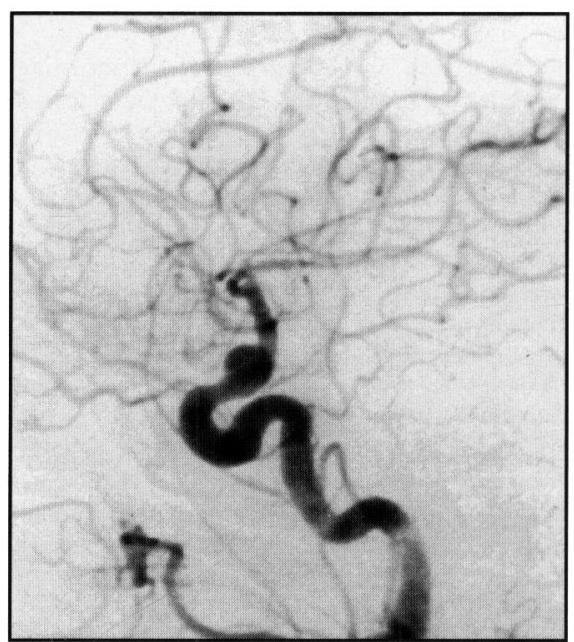

Figure 4: Patient 1. AP $(\boldsymbol{A})$ and lateral $(\boldsymbol{B})$ angiograms demonstrating a small, dorsomedially projecting aneurysm arising distally on the left internal carotid artery, between the PCOA and AChA. A small secondary bleb arising from the aneurysm body is seen inferiorly on the AP view, indicating the site of rupture. 
A

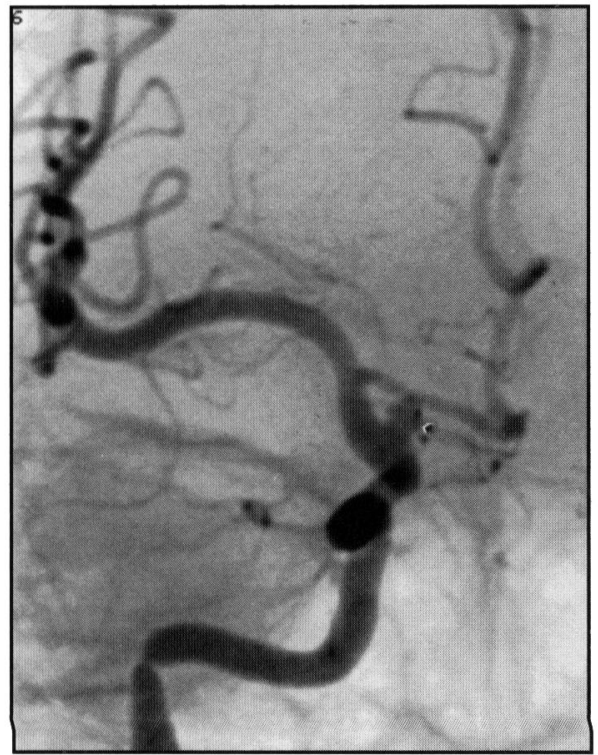

C

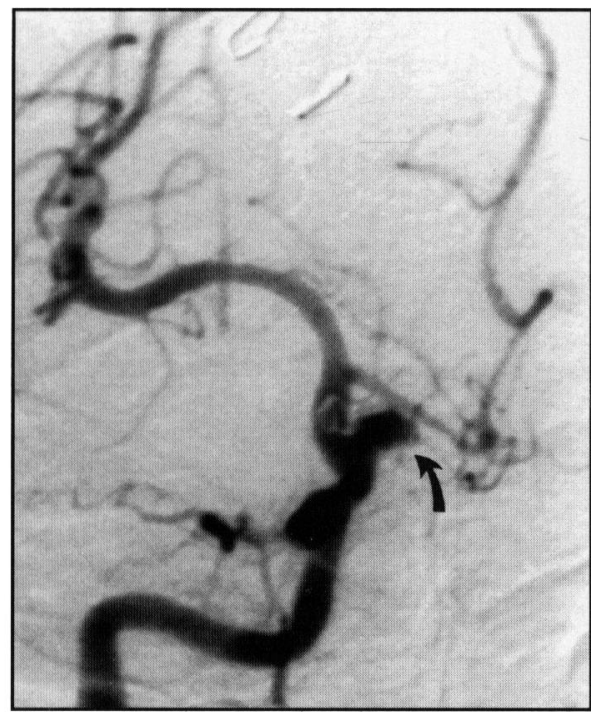

B

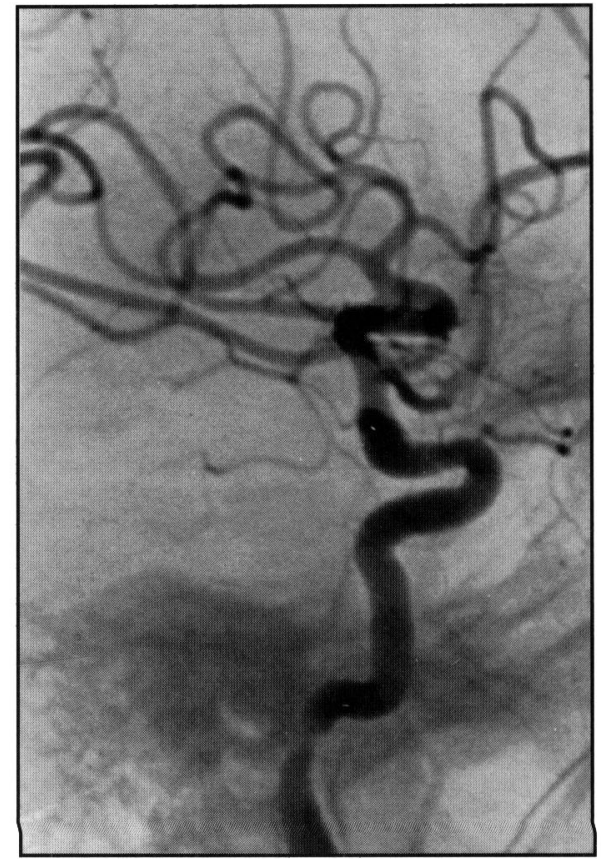

D

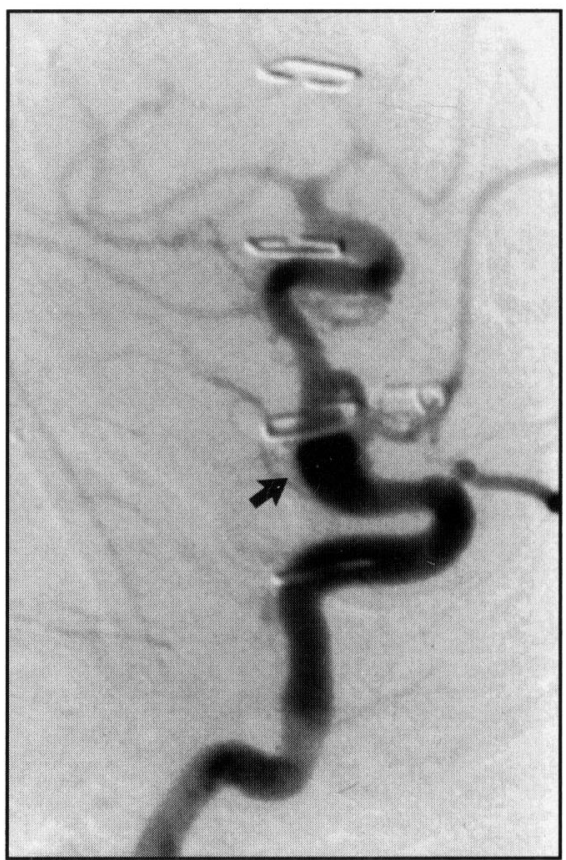

Figure 5: Patient 2. AP $(A)$ and lateral $(B)$ angiograms demonstrating a small, medially projecting aneurysm arising distally on the right internal carotid artery, between the PCOA and AChA. The aneurysm was clipped uneventfully. Three days later the patient deteriorated abruptly from recurrent hemorrhage. Repeat angiography demonstrated a new aneurysm immediately beneath the clip blades (arrows, $C$ and $D$ ). The new aneurysm is slightly larger and more medially directed than the original. A false aneurysm was found at re-exploration.

elastic membrane lead to aneurysm growth. Interruptions in the medial layer at arterial branch points have been described, but a contribution of these "medial defects" to aneurysm formation is not universally accepted. ${ }^{14}$ Both the arterial media and internal elastic laminae must be absent or structurally weakened for an aneurysm to develop, and when examined histologically, the media usually terminates abruptly at the aneurysm neck. ${ }^{13}$ Occlusion of a major cerebral vessel may increase the possibility of aneurysm formation at large arterial forks subjected to the augmented hemodynamic stress associated with collateral flow. ${ }^{14}$

A possible contribution of atherosclerosis to saccular aneurysm formation has been suggested by Stehbens. ${ }^{14}$ Loss of tensile strength and decreased cohesive properties of the vessel wall tissue may lead to arterial dilatation or outpouching. It is interesting to note that atherosclerotic changes in the preaneurysmal segment of the ICA have been a frequent but inconstant observation in those cases of distal ICA aneurysms which 
are unrelated to arterial bifurcations. 8,10 The internal carotid artery has a gentle medial to posterolateral curve as it exits the cavernous sinus, with maximum hemodynamic thrust therefore against the dorsomedial distal ICA wall. This mechanical stress, possibly combined with degenerative atherosclerotic tissue weakening, may be enough to cause aneurysm formation even without the typical hemodynamic factors and deficiency of the media related to arterial bifurcations. An atypical mechanism of formation may also account for the unusually thin and friable aneurysm necks which make these aneurysms so prone to intraoperative bleeding. ${ }^{10}$

\section{Surgical Considerations}

Internal carotid artery aneurysms which arise distally on the dorsomedial wall have an unusually high risk of intra-operative rupture $^{8-10}$ and are associated with surgical risks that are disproportional to their relative lack of anatomical complexity. Dissection of the sylvian fissure and exposure of the aneurysm neck must be done with minimal frontal lobe retraction, especially in the case of a superiorly projecting aneurysm which might be adherent to or embedded in the parenchyma. Wide opening of the sylvian fissure is necessary to allow proximal and distal control of the internal carotid artery, and to permit clip placement from the most favorable trajectory.

Ideally, a clip is best placed on the aneurysm neck in parallel to the parent artery, or at a slightly oblique angle, rather than perpendicular. Temporary occlusion of the ICA at the time of clipping to decrease turgor and minimize wall stress will reduce the risk of neck avulsion as the blades are closed. Tearing of the aneurysm neck at the time of clipping or post-operatively has been a significant complication in the treatment of these aneurysms. Two in the current series tore at the neck at the time of clipping, and one rebled on the third post-operative day, with formation of a false aneurysm. Yasargil ${ }^{6}$ described his experience with two aneurysms of this type, one of which ruptured at the base during dissection. The internal carotid artery was repaired directly with micro sutures. Shigeta et al. ${ }^{10}$ reported on 20 dorsal internal carotid aneurysms, with intra-operative rupture in five cases, four as a result of laceration at the neck. Two other cases had post-operative hemorrhage which was attributed to new growth of the aneurysm due to insufficient inclusion of the wall of the parent artery within the clip blades. Reinforcement of the clip and parent artery with cellulose fabric to prevent slipping and to reinforce the fragile wall was recommended.

The potential for intra-operative rupture must be anticipated, and preparations made for proximal control. Exposure of the carotid artery in the neck adds little time to the procedure and provides an extra measure of security during craniotomy and initial dissection. Angiographic assessment of collateral supply to the ipsilateral hemisphere pre-operatively will assist in determining the safety of temporary or deliberate permanent ICA occlusion. A drawback to aneurysm trapping is that the neck is usually distal to the PCoA, and occlusion distal to this branch renders hemispheric blood flow dependent solely on the anterior communicating artery and leptomeningeal collaterals. If there is any doubt about cerebrovascular reserve, emergency revascularization with a superficial temporal artery or saphenous vein bypass may be considered.
Because of their unusually high surgical risk, alternative methods of treatment merit consideration. Unfortunately, dorsomedial carotid aneurysms are usually broad-based with a relatively wide-neck, an anatomical characteristic which significantly reduces the likelihood of satisfactory obliteration with electrolytically detachable coils. ${ }^{15}$ However, because they are unrelated to major arterial branches, these aneurysms may be particularly well suited to developing endovascular techniques employing a combination of stent implantation or balloon remodeling and coil packing. ${ }^{16.17}$

In conclusion, an uncommon but anatomically distinct subtype of supraclinoid carotid aneurysm is described. These aneurysms do not conform to the usual principles of aneurysm formation and are unique in their dorsomedial location unrelated to an arterial bifurcation. Although their anatomy is straightforward and exposure is not restricted by bone or dural structures of the skull base, these aneurysms possess extremely fragile necks which make surgical management particularly hazardous.

\section{REFERENCES}

1. Gibo H, Lenkey C, Rhoton AL. Microsurgical anatomy of the supraclinoid portion of the internal carotid artery. J Neurosurg 1981; 55: 560-574.

2. Rhoton AL. Anatomy of saccular aneurysms. Surg Neurol 1980; 14: 59-66.

3. Batjer HH, Kopitnik TA, Giller CA, Samson DS. Surgery for paraclinoidal carotid artery aneurysms. J Neurosurg 1994; 80: 650658.

4. Day AL. Aneurysms of the ophthalmic segment: a clinical and anatomical analysis. J Neurosurg 1990; 72: 677.691.

5. Knosp E, Müller G, Perneczky A. The paraclinoidal carotid artery: anatomical aspects of a microneurosurgical approach. Neurosurgery 1988; 22: 896-90I.

6. Yasargil MG. Microneurosurgery, Vol II: Clinical Considerations, Surgery of the Intracranial Aneurysms and Results. New York: Georg Thieme Verlag, 1984; 33-123.

7. Aldrich F. Anterior (dorsal) paraclinoid aneurysm: case report. Surg Neurol 1991; 35: 374-376.

8. Ishikawa T, Nakamura N, Houkin K, Nomura M. Pathological consideration of a "blister-like" aneurysm of the superior wall of the carotid artery: case report. Neurosurgery 1997; 40: 403-406.

9. Nakagawa F, Kobayashi S, Takemae T, Sugita K. Aneurysm protruding from the dorsal wall of the internal carotid artery. J Neurosurg 1986; 65: 303-308.

10. Shigeta $\mathbf{H}$, Kyoshima K, Nakagawa F, Kobayashi S. Dorsal internal carotid artery aneurysms with special reference to angiographic presentation and surgical management. Acta Neurochir (Wien) 1992; 119: 42-48.

11. Yoshimoto Y, Ochiai C, Nagai M. Cerebral aneurysms unrelated to arterial bifurcations. Acta Neurochir (Wien) 1996; 138: 958-964.

12. Ferguson GG. Physical factors in the initiation, growth, and rupture of human intracranial saccular aneurysms. J Neurosurg 1972; 37: 666-677.

13. Sekhar LN, Heros RC. Origin, growth, and rupture of saccular aneurysms: a review. Neurosurgery 1981; 8: 248-260.

14. Stehbens WE. Etiology of intracranial berry aneurysms. J Neurosurg 1989; 70: 823-831.

15. Zubillaga AF, Gugliemi G, Viñuela F, Duckwiler GR. Endovascular occlusion of intracranial aneurysms with electrically detachable coils: correlation of neck size with treatment results. Am J Neuroradiol 1994; $15: 815-820$.

16. Szikora I, Guterman LR, Wells KM. Hopkins LN. Combined use of stents and coils to treat experimental wide-necked carotid aneurysms: preliminary results. Am J Neuroradiol 1994; 15:1091-1102.

17. Mericle RA, Wakhloo AK, Rodriguez $\mathrm{R}$, et al. Temporary balloon protection as an adjunct to endosaccular coiling of wide-necked cerebral aneurysms: technical note. Neurosurgery 1997; 41: 975-978. 